\title{
Study on Characteristics of Indica Rice Price Fluctuation in China Based on GARCH model
}

\author{
Jianlin Peng, Xuerong Xu \\ College of Economics, Fujian Agriculture and Forestry University, Fuzhou, Fujian Province, China.
}

Keywords: price of indica rice; GARCH model; fluctuation analysis; clustering.

\begin{abstract}
The GARCH model is used to analyze fluctuations in the earning price ratio of indica rice in China from August 1998 to July 2017. The study shows that, the residual sequence of indica rice earning price ratio has a high order ARCH effect and the characteristic of volatility clustering; it does not have the significant feature of high risk and high return. Price fluctuations in the past have slow and spreading effects on current price. External factors have symmetrical impacts on the price of early indica rice, but the impacts are asymmetrical on late indica rice. Negative impacts can cause more price fluctuations in next period.
\end{abstract}

\section{Introduction}

Since 2004, the grain price safeguard system which is represented by the minimum purchase price policy has played a positive role in maintaining grain production and increasing farmers' income. But to a certain extent, it prevents the market price of grains from adjusting the relationship between supply and demand. In recent years, domestic agriculture has developed steadily; the domestic price of rice has gradually exceeded the international price. Domestic rice price is now facing a great downward pressure. In the future, the policy of minimum purchase price of rice will be cancelled; rice farmers will be greatly impacted by domestic and international markets. In this paper, an empirical study is carried out to analyze fluctuations in prices of early and late indica rice in China through GARCH model. The author also explores the characteristics of volatility, asymmetry as well as high risk and high return, so as to provide reference for the scientific prediction of rice price fluctuation risks.

\section{ARCH Model and Setting}

\subsection{ARCH model.}

In 1982, Engle proposed the auto regressive conditional heteroscedasticity (ARCH) model. The model uses conditional heteroscedasticity to explain differences of fluctuation sizes in sequences at different periods, and uses autoregression to explain the relationship between fluctuations. The ARCH model goes as following.

$$
\begin{array}{r}
Y_{\mathrm{t}}=X_{\mathrm{t}}{ }^{\prime} \beta+\varepsilon_{\mathrm{t}} \\
h_{t}=\alpha_{0}+\sum_{j=1}^{q} \alpha_{i} \varepsilon_{t-i}^{2}
\end{array}
$$

Formula (1) is the mean value equation. $\mathrm{Y}$ is the interpreted variable; $\mathrm{X}$ is the explanatory variable; $\varepsilon$ is a random disturbance term. Formula (2) is the variance equation. $h$ is the conditional variance of $\varepsilon ; \alpha$ is the parameter.

\subsection{GARCH model.}

In 1986, Bollerslev proposed a generalized auto regressive conditional heteroscedasticity model 
(GARCH) to solve the problem of higher value of autoregressive process order ${ }^{q}$ in some time series analyses. The variance equation in the ARCH model is modified as: $h_{t}=\alpha_{0}+\sum_{j=1}^{q} \alpha_{i} \varepsilon_{t-i}^{2}+\sum_{j=1}^{\mathrm{p}} \theta_{i} h_{t-i}$. In which, $\sum_{j=1}^{q} \alpha_{i} \varepsilon_{t-i}^{2}$ is the ARCH (q) term, $\sum_{j=1}^{\mathrm{p}} \theta_{i} h_{t-i}$ is the ARCH $(p)$ term, $p, \theta$ are lag orders respectively. In practice, the order value $q$ of GARCH is much smaller than that of ARCH model. In general, the GARCH $(1,1)$ model can portray most of the financial time series data.

\subsection{GARCH-M model.}

In order to explain the rates of return on investment of financial assets, Engle and his colleagues added the conditional variance $h$ to the mean value equation of GARCH model. The mean equation becomes $Y_{\mathrm{t}}=X_{\mathrm{t}}{ }^{\prime} \beta+\rho h_{\mathrm{t}}+\varepsilon_{\mathrm{t}}$. Conditional variance $h$ represents the size of expected risk. When its coefficient $\rho$ is significantly greater than zero, assets are considered to have the feature of high risk and high return.

\subsection{T-GARCH model.}

In the GARCH model, conditional variance $h$ is determined by the absolute value of residuals, and has nothing to do with its positive or negative relationship. Fluctuations in asset prices tend to be different when prices rise and fall. Experience shows that price fluctuations are usually larger when price decreases. That is to say, the impacts of external market information on fluctuation are asymmetric. The T- GARCH model explains that kind of asymmetry by adding virtual variables. In that case, the conditional variance is: $h_{t}^{2}=\alpha_{0}+\sum_{j=1}^{q} \alpha_{i} \varepsilon_{t-i}^{2}+\sum_{j=1}^{\mathrm{p}} \theta_{i} h_{t-i}^{2}+\gamma \varepsilon_{t-1}^{2} d_{t-1}$, in which $d_{t}$ is a virtual variable which satisfies $d_{t}=\left\{\begin{array}{ll}1, & \varepsilon_{t}<0 \\ 0, & \varepsilon_{t}>0\end{array}\right.$. When $\gamma>0$, negative impacts impose more influences on fluctuation than positive impacts. When $\gamma<0$, negative impacts impose fewer influences on fluctuation than positive impacts.

\section{Sample and Data Description}

The price data of early indica rice (ER) and late indica rice (LR) in the national wholesale market from July 1998 to July 2017 were collected from the China Agricultural Information Network. The logarithmic return rate formula $r_{t}=\ln P_{t}-\ln P_{t-1}$ is used to analyze the price volatility. In the formula, $r_{t}$ is the price of rice in the tth period; $P_{t} 、 P_{t-1}$ are the prices of rice in the th and $t$ - 1 th period respectively. The earnings price ratio fluctuates around the value of " 0 "; the yield rate appears continuously above or under 0 , showing a certain degree of volatility clustering. According to statistical index, the kurtosis of indica rice earnings price ratio is far greater than that of the standard normal distribution, showing it has the characteristics of financial time series of "obvious peak and fat tails". From the J-B statistics, it can be seen that both values are relatively large and different from the normal distribution.

Table 1 Statistics description of earnings price ratio of indica rice

\begin{tabular}{lccccccc}
\hline & & & & standard & & J-B \\
breed & mean & maximum & minimum & deviation & skewness & kurtosis & value \\
\hline Early indica & & & & & & & \\
rice & $0.33 \%$ & $16.85 \%$ & $-8.75 \%$ & $2.26 \%$ & 15.26 & 1.57 & 3249 \\
Late indica rice & $0.26 \%$ & $16.74 \%$ & $-8.46 \%$ & $2.29 \%$ & 20.45 & 2.67 & 5746 \\
\hline
\end{tabular}




\section{Establishing the Model of Indica Rice Earning Price Ratio and Volatility Analysis}

The price logarithmic yield stability of early indica rice and late indica rice were tested to avoid pseudo regression caused by the uneven model. The results of ADF test showed that the earnings price ratios of early and late indica rice were -4.8325 and -7.4705 respectively, less than the significant level of $1 \%$; the logarithmic yield rates of early and late indica rice were stable. The autocorrelation and partial autocorrelation analysis on the price logarithmic yield rate of early indica rice showed that the value of $\mathrm{Q}$ statistics was greater than the significant level of $1 \%$; there is correlation in earnings price ratio. SC and AIC minimum criteria are used to determine ARIMA $(2,2)$ and ARIMA $(4,2)$ as the mean value equation of earnings price ratio of early and late indica rice.

$$
\begin{aligned}
& \text { Erice }_{\mathrm{t}}=0.002134 \text { Erice }_{\mathrm{t}-1}+0.490165 \text { Erice }_{\mathrm{t}-2}+0.187300 \varepsilon_{\mathrm{t}-1}-0.256152 \varepsilon_{\mathrm{t}-2} \\
& \text { Lrice }_{\mathrm{t}}=0.312845 \text { Lrice }_{\mathrm{t}-4}+0.233388 \varepsilon_{\mathrm{t}-1}+0.168508 \varepsilon_{\mathrm{t}-2}
\end{aligned}
$$

In order to find out whether there is heteroscedasticity in earning price ratio, the residual term square of the mean value equation is tested by ARCH-LM. The results show that, when the lag order is 4, the F statistical magnitude of early indica rice earnings price ratio is 5.92 with $\mathrm{P}$ value of 0.0002 ; the F statistical magnitude of late indica rice is 11.58 with $\mathrm{P}$ value of 0.00 . It can be seen that there is a high order ARCH effect in the residual squared term of the mean equation. In order to avoid overestimating parameters, a low order GARCH is used to build the variance equation model. According to AIC minimum and model coefficient requirements, GARCH $(1,1)$ and GARCH $(2,1)$ are selected to build the model of price logarithmic yield rate of early and late indica rice. The results are as following.

\begin{tabular}{|c|c|c|c|c|c|c|c|c|c|}
\hline \multirow{2}{*}{$\begin{array}{l}\text { Distri-b } \\
\text { ution } \\
\text { model }\end{array}$} & \multicolumn{3}{|c|}{ Normal distribution } & \multicolumn{3}{|c|}{ Student-t distribution } & \multicolumn{3}{|c|}{ GED distribution } \\
\hline & $\begin{array}{l}\text { GARC } \\
\mathrm{H}(1,1)\end{array}$ & $\begin{array}{l}\text { GARCH } \\
(1,1)-M\end{array}$ & $\begin{array}{l}\text { TGARC } \\
\mathrm{H}(1,1)\end{array}$ & $\begin{array}{l}\text { GARC } \\
\mathrm{H}(1,1)\end{array}$ & $\begin{array}{l}\text { GARCH } \\
(1,1)-M\end{array}$ & $\begin{array}{l}\text { TGARC } \\
\mathrm{H}(1,1)\end{array}$ & $\begin{array}{l}\text { GARCH } \\
(1,1)\end{array}$ & $\begin{array}{l}\text { GARCH } \\
(1,1)-M\end{array}$ & $\begin{array}{l}\text { TGARC } \\
\mathrm{H}(1,1)\end{array}$ \\
\hline $\mathrm{AR}(1)$ & -1.210 & $-1.249^{* * *}$ & $-1.176^{* * *}$ & -0.362 & $-0.584^{* *}$ & -0.265 & -0.025 & -0.053 & -0.027 \\
\hline $\mathrm{AR}(2)$ & -0.582 & $-0.630^{* * *}$ & $-0.592^{* * *}$ & 0.108 & 0.106 & 0.057 & 0.025 & 0.001 & 0.048 \\
\hline MA(1) & $1_{* * *}^{1.308}$ & $1.335^{* * *}$ & $1.291^{* * *}$ & 0.472 & $0.674^{* *}$ & 0.378 & 0.094 & 0.101 & 0.094 \\
\hline MA(2) & $\underset{* * *}{0.748}$ & $0.785^{* * *}$ & $0.756^{* * *}$ & 0.065 & 0.048 & 0.109 & $0.239^{* *}$ & $0.264^{* * *}$ & $0.222^{*}$ \\
\hline$\rho$ & & $0.137^{*}$ & & & 0.077 & & & 0.009 & \\
\hline$a_{0}$ & ${ }_{* * *}^{0.0001}$ & $0.000^{* * *}$ & $0.000^{* * *}$ & 0.000 & 0.000 & 0.000 & 0.000 & 0.000 & 0.000 \\
\hline$a_{1}$ & 0.389 & $0.366^{* * *}$ & $0.327^{* * *}$ & 0.548 & 0.577 & 0.483 & 0.312 & 0.307 & 0.314 \\
\hline$\theta_{1}$ & $\underbrace{0.458}_{* * *}$ & $0.528^{* * *}$ & $0.545^{* * *}$ & $0.410^{*}$ & $0.364^{*}$ & $0.401^{* *}$ & 0.437 & 0.427 & 0.432 \\
\hline $\begin{array}{l}\gamma \\
\text { GED }\end{array}$ & & & 0.124 & & & 0.435 & $0.815^{* * *}$ & $0.815^{* * *}$ & $\begin{array}{l}-0.011 \\
0.807^{* * *}\end{array}$ \\
\hline AIC & -4.993 & -5.000 & -5.000 & -5.23 & -5.22 & -5.23 & -5.22 & -5.21 & -5.21 \\
\hline loglike & 568.77 & 570.10 & 568.83 & 596.42 & 596.66 & 596.67 & 594.86 & 594.19 & 594.76 \\
\hline
\end{tabular}

Table 2 Comparison of GARCH earning price ratios of early indica rice under different distributions

Note: $* * *$ represents $1 \%$ significance level, ** represents $5 \%$ significance level, * represents $10 \%$ significance level, GED represents parameters of GED distribution, loglike is the likelihood value. Similarly hereinafter

Based on AIC maximum value and likelihood maximum value, it can be inferred that the GARCH model with residuals under Student-t distribution can better reflect fluctuations of earning price ratio of early indica rice. All parameters in the variance equation of early indica rice price logarithm yield are positive, showing that clustering exists in the fluctuation of early indica rice price. Fluctuations in 
last period has positive impacts on fluctuations in next period; larger fluctuations are followed by longer ones. The sum of $a 0, a 1$ and $\theta_{1}$ is between 0.884 and 0.958 ; the influence of past fluctuations will slowly disappear. $\rho$ is not significantly greater than 0 . There is no feature of high risk and high return in the market of early indica rice. The coefficient of TGARCH $(1,1)$ model is not significant, showing the weak asymmetry in earnings price ratio of early indica rice.

For late indica rice, evaluate from the smallest AIC value and largest likelihood value, the Student-t distribution and GED distribution are obviously more suitable. The GARCH model of GED distribution can be used to analyze the price fluctuation of late indica rice with parameter significance level as reference. The price fluctuation of late indica rice also has the clustering characteristic. The price fluctuation in previous period has positive effects on the price of this period. The sum of a0, a1, $\theta_{1}$ and $\theta_{2}$ in GARCH $(2,1)$-M model is between 0.8981and 0.953; the influence of past fluctuations will vanish slowly. $\rho$ is not significant; the late indica rice market does not have the feature of high risk and high return, either. The coefficient of dummy variable in TGARCH $(2,1)$ model is greater than 0 ; negative impact will cause greater price fluctuations in next period.

Table 3 Comparison of GARCH earning price ratios of late indica rice under different distribution

\begin{tabular}{|c|c|c|c|c|c|c|c|c|c|}
\hline \multirow{2}{*}{$\begin{array}{l}\begin{array}{l}\text { Distri- } \\
\text { bution }\end{array} \\
\text { model }\end{array}$} & \multicolumn{3}{|c|}{ Normal distribution } & \multicolumn{3}{|c|}{ Student-t distribution } & \multicolumn{3}{|c|}{ GED distribution } \\
\hline & $\begin{array}{l}\text { GARCH } \\
(2,1)\end{array}$ & $\begin{array}{l}\text { GARCH } \\
(2,1)-M\end{array}$ & $\begin{array}{l}\text { TGARC } \\
\mathrm{H}(2,1)\end{array}$ & $\begin{array}{l}\text { GARCH } \\
(2,1)\end{array}$ & $\begin{array}{l}\text { GARCH } \\
(2,1)-M\end{array}$ & $\begin{array}{l}\text { TGARC } \\
\mathrm{H}(2,1)\end{array}$ & $\begin{array}{l}\text { GARC } \\
\mathrm{H}(2,1)\end{array}$ & $\begin{array}{l}\text { GARCH } \\
(2,1)-M\end{array}$ & $\begin{array}{l}\text { TGARC } \\
\mathrm{H}(2,1)\end{array}$ \\
\hline $\mathrm{AR}(4)$ & 0.1875 & $0.1810^{* * *}$ & $0.1496^{* *}$ & $0.1114^{* *}$ & $0.1113^{* *}$ & $0.0912^{*}$ & $0.0873^{* *}$ & $0.1069^{* *}$ & $0.0802^{*}$ \\
\hline $\operatorname{MA}(1)$ & 0.4274 & $0.4315^{* * *}$ & $0.4552^{* *}$ & $0.2347^{* *}$ & $0.2343^{* * *}$ & $0.2312^{* * *}$ & $0.1838^{* *}$ & $0.1830^{* * *}$ & $0.1877^{* * *}$ \\
\hline $\operatorname{MA}(2)$ & 0.2355 & $0.2407^{* * *}$ & $0.2426^{* * *}$ & $0.1683^{* *}$ & $0.1681^{* * *}$ & $0.1650^{* * *}$ & $0.1594^{* *}$ & $0.1644^{* * *}$ & $0.1598^{* * *}$ \\
\hline$\rho$ & & $-1.6231^{* *}$ & & & 0.0256 & & & 0.7646 & \\
\hline $\mathrm{a}_{0}$ & $0.0000^{*}$ & $0.0000^{*}$ & $0.0000^{* *}$ & 0.0001 & 0.0001 & 0.0001 & 0.0000 & 0.0000 & 0.0000 \\
\hline$a_{1}$ & $\underset{* * *}{0.9143}$ & $0.9231^{* * *}$ & $0.6459^{* *}$ & 0.5066 & 0.5065 & 0.2388 & $0.4351^{* *}$ & $0.4559^{* * *}$ & $0.3947^{* * *}$ \\
\hline$\theta_{1}$ & $0.1081^{*}$ & $0.1003^{*}$ & $0.0394^{* *}$ & 0.3189 & 0.3202 & 0.4777 & 0.2540 & 0.2493 & 0.2267 \\
\hline$\theta_{2}$ & 0.2799 & $0.2723^{* * *}$ & $0.3182^{* *}$ & 0.1991 & 0.1986 & 0.1252 & 0.2639 & 0.2565 & 0.2767 \\
\hline $\begin{array}{l}\gamma \\
\text { GED }\end{array}$ & & & $0.7860^{* *}$ & & & 0.5388 & $0.8911^{* *}$ & $0.8946^{* * *}$ & $\begin{array}{l}0.3301 \\
0.9080^{* * *}\end{array}$ \\
\hline AIC & -5.2691 & -5.2640 & -5.2743 & -5.4822 & -5.4732 & -5.4845 & -5.4509 & -5.4425 & -5.4462 \\
\hline loglike & 597.51 & 594.93 & 596.09 & 619.26 & 620.52 & 619.26 & 615.78 & 615.84 & 616.25 \\
\hline
\end{tabular}

\section{Conclusions and Policy Recommendations}

\subsection{Conclusions.}

The price fluctuations of early and late indica rice are analyzed through constructing GARCH models and get following conclusions. The earning price ratios of early and late indica rice have non normal characteristics of sharp peak and fat tails. The earnings price ratios of both early and late indica rice are right-skewed. Comparatively speaking, the skewness and kurtosis of late indica rice are greater than those of early rice. Volatility clustering exist in both species. The impacts of past volatility on earning price ratio will slowly disappear, and there is no feature of high risk and high return. The impact of external positive factors on early indica rice market is greater than negative factors; the asymmetry of late indica rice market is weaker than early rice. 


\subsection{Policy recommendations.}

Late indica rice has the largest yield in all kinds of rice; it is the main body of residents' rice consumption. Its overall distribution of earning price ratio is more steep. Thus, more attention should be paid to the monitoring of late indica rice price. As earlier fluctuations has long lasting impacts on earning price ratio, appropriate measures should be taken to absorb these effects. The price of indica rice is not characterized by high risk and high return, partly because the current minimum grain purchase price serves as the floor. With the establishment of grain target price and the gradual withdrawal of minimum purchase price policy, the relationship between rice import and domestic rice production should be coordinated to formulate reasonable and effective target prices of rice.

\section{Acknowledgement}

Fund Project: This paper is one of the outcomes of the research, The Determination of Rice Planting Income Insurance Rates in Different Regions of China and Its Impacts on Farmers' Purchase Intention, which is supported by the Training Programme Foundation for Young Talents of Fujian Province in 2015. (Project No.: Document of Research Programmes in Colleges and Universities of Fuzhou, [2016] No. 25).

\section{References}

[1] F.N. Zhong, Can grain reserve and price control stabilize the grain market? -- Some enlightenment from the world food crisis, J. Journal of Nanjing Agricultural University (Social Science Edition). 2 (2011).

[2] L. Sun, K.K. Ni, Asymmetric analysis of international grain price fluctuation: based on EGARCH model under T distribution, J. Journal of Nanjing Agricultural University (Social Science Edition). 2 (2013).

[3] G.H. Lin, T. Chen, Empirical analysis of international rice price fluctuation: based on ARCH model, J. Chinese Rural Economy. 2 (2011).

[4] L. Zhan, H.P. Jiang, Predicaments in the reform of grain target price system and breakthrough, J. Issues in Agricultural Economy. 2 (2015).

[5] L. Xu, Q. Zhang, Risk assessment on market price of grains in China, J. Journal of China Agricultural University. 16 (2011).

[6] R.F. Engle, Autoregressive conditional heteroscedasticity with estimates of the variance of United Kingdom inflation, J. Econometrica. 50 (1982).

[7] T. Bollerslev, Generalized autoregressive conditional heteroskedasticity, J. Journal of Econometrics. 31 (1986). 